\title{
OPPORTUNITIES IN DOING THE GREATER GOOD: A BIGGER ROLE FOR PHARMA
}

Dear Reader,

While the Indian Pharmaceutical Industry has earned recognition across the globe for cost-effective, high quality pharmaceuticals, controlling the prevalence of even well-treatable diseases in India has not progressed nearly as admirably. A study of the global burden of disease recently published in The Lancet by the Institute for Health Metrics and Evaluation at the University of Washington ranked India's healthcare access and quality (HAQ) at a low 154 among 195 countries.

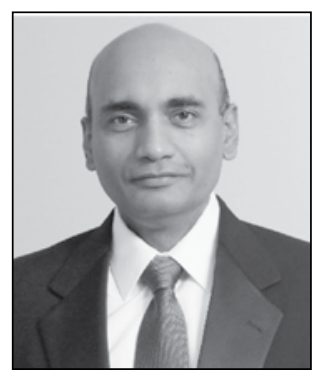
$H A Q$ index for India was 45, worse than Nepal (51), Bangladesh (52) and Sri Lanka (73). For tuberculosis treatment, India scored 26 out of 100, less than Congo (30), Djibouti and Pakistan (29 for both). The HAQ score for neonatal mortality in India was 14 out of 100, worse than Afghanistan (19) or Somalia (21). India's HAQ scores for chronic kidney diseases and diabetes were 20 (rank 176) and 38 (rank 151), respectively.

With a fast growing pharmaceutical industry that has captured greater than 20 and 40 percent of European and American prescription volumes respectively, and a health care industry that is attracting a significant fraction of global medical tourism, it is unbecoming for a rising global power like India to sport health metrics that belie one of the fastest growing economies in the world. Given the centrality of health in economic development, a paradigm shift in addressing the issue is needed. It may be easy to point fingers at deficiencies in the Public Health System, which may be starting to make inroads with the new no cost health insurance for the poor rolled out in 2018, but the program's ability to address the issue is not a given. Persistent difficulties in public health care system such as poor administration of resources, corruption, lack of accountability and fraud can easily scuttle even the best of the initiatives. Given the size and socioeconomic diversity of India, the complexity of the issue or its scale cannot be underestimated but that can present an opportunity by its very nature.

Perhaps second only to IT industry's growth, Indian pharma industry has grown by leaps and bounds over the past few decades. It has the opportunity to expand its role in domestic health well beyond simply manufacturing and selling, by leveraging its intimate understanding of the Indian pharmaceutical market. Ways in which the pharma industry can play a bigger vital role in national health can include: disease management in collaboration with individual physicians and/or hospital systems; sponsorship of health care camps to increase awareness of silent diseases such as diabetes and cardiovascular ailments to help undiagnosed patients seek care; supporting affordable medicines for low income patients; supporting academic-industrial collaboration in areas of strategic importance; and, supporting open source public health innovations. Efforts in any of these directions can be a mutually beneficial three-way win for the public, the nation and the industry.

Taking an example, compared to about 40 million in China and 30 million in the United States, Type 2 diabetes is predicted to affect nearly 80 million people in India by 2030, all who are at risk of amputated limbs, blindness, heart disease and other serious complications. Supporting disease management in the area with a full portfolio of products can benefit a huge swath of population and present the opportunity for a company to emerge with a reputation for being a disease specialist. Efforts expended in this direction can lead to better understanding of the market segments of patient populations, their needs and the optimal ways to treat them. Real world data collected in the process can 
also be far more valuable than data collected from tightly controlled clinical trials on highly selective populations selected based on strict inclusion/exclusion criteria. Real world data can be faster and less expensive to collect, be more discriminatory against poorly/marginally effective treatments, and safely allow for the inclusion of and the understanding of high risk patient groups. With the decreasing cost of data collection and data analysis and given the emerging regulatory trends/guidances that are starting to shine positive light on real world data, the timing is right for enterprising organizations to move in this direction. Real World Evidence can be used to supplant expensive randomized controlled clinical trials, opening up new opportunities for enterprises that are customer/disease-focused. Insights, be it a marketing insight, a clinical insight or disease insight, gained from such a venture can also translate better in the global markets.

With the growth of internet and social media, patients today have access to far more medical information than ever before. An increasing cross section of patients is far more knowledgeable about disease, treatment options and own needs than in the past. Globally, as healthcare increasingly transitions toward patient-driven choice that is focused on outcomes and value, episodic interaction with the patient needs to evolve into more interactive ways to deliver ongoing care with better understanding of external factors such as behavior, social circumstances and environmental influences. Enterprises focused on doing the greater good by focusing on the needs of patients and the society at large will have the ability to capitalize and thrive on the emerging trends in the regulatory and market landscape. It is not too late to begin or expand.

\section{About The Guest Editor}

Dr. Jay Lakshman is Director/Global Technical Project Management Leader guiding the development of portfolio projects at Novartis Pharmaceuticals Corporation in US. He has been responsible for numerous INDs and three successfully launched commercial products. He has over 10 patents and 40 presentations/publications and is recognized as Novartis Leading Scientist for his work in enabling development of difficult drug candidates. He received his Ph.D. in Drug Delivery from Rutgers-The State University of New Jersey, Masters from Indian Institute of Technology-BHU and Bachelors from Madurai Medical College.

4.

If you would like to comment on the Guest editorial please write to us at publications@idmaindia.com 